Pis'ma v ZhETF

\title{
Testing the correlations between ultra-high-energy cosmic rays and BL Lac type objects with HiRes stereoscopic data
}

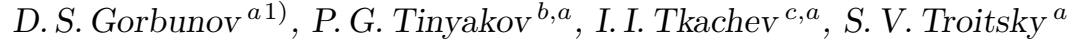 \\ ${ }^{a}$ Institute for Nuclear Research of the Russian Academy of Sciences, \\ 60th October Anniversary Prospect 7a, 117312, Moscow, Russia; \\ ${ }^{b}$ Service de Physique Théorique, CP 225, Université Libre de Bruxelles, B-1050, Brussels, Belgium; \\ ${ }^{c}$ CERN Theory Division, CH-1211 Geneva 23, Switzerland.
}

\begin{abstract}
Previously suggested correlations of BL Lac type objects with the arrival directions of the ultra-highenergy cosmic ray primaries are tested by making use of the HiRes stereoscopic data. The results of the study support the conclusion that BL Lacs may be the cosmic ray sources and suggest the presence of a small (a few percent) fraction of neutral primaries at $E>10^{19} \mathrm{eV}$.
\end{abstract}

'PACS: 98.70.Sa, 98.54.Cm

\section{MOTIVATION}

With the exception of a small number of highestenergy events, the bulk of ultra-high energy cosmic rays (UHECR) is most naturally explained by acceleration in active galaxies [1, 2, 3]. This explanation is supported 'by clustering of UHECR observed in the AGASA and Yakutsk datasets 4, 5, 6 ${ }^{2)}$ and by correlations between arrival directions of UHECR and a particular class of active galactic nuclei, the BL Lacertae objects (BL Lacs) 11, 12, 13] (see also Ref. 14).

The correlations with BL Lacs were observed in AGASA and Yakutsk datasets. To test them, independent datasets are needed. Such a dataset has been recently published by the HiRes collaboration [8]. It consists of 271 events with reconstructed energy $E>$ $10^{19} \mathrm{eV}$ which were observed in the stereoscopic mode. The energies of the events are not published; this makes reconstruction [12] of arrival directions in the Galactic magnetic field impossible. Despite the small exposure of HiRes in the stereo mode as compared to AGASA, the excellent angular resolution of $0.6^{\circ}$ makes HiRes data quite competitive (or even superior) in testing correlations with BL Lacs under the assumption of neutral primary particles. This test is the purpose of the present work. More precisely, we take three previously studied sets of BL Lacs without any further selection and test them for correlations with the entire set of 271 HiRes events.

\footnotetext{
1) e-mail: gorby@ms2.inr.ac.ru

${ }^{2)}$ HiRes collaboration does not observe significant clustering 78 but, at the present level of statistics, this does not contradict to the previous results 9 [10].
}

\section{FLUCTUATIONS OF CORRELATION SIGNAL}

At the current low statistics the correlation signal is not expected to be robust [5]. To illustrate this important point consider the following example. Assume that a given set of sources in a given exposure time produces 5 events in average. Let the average number of rays coincident with sources by chance be 2 . The average number of correlating rays observed in such an experiment would therefore be 7 . Both the real events from sources and background chance coincidences are Poisson distributed. Thus, in a particular realization the number of correlating rays would vary roughly within $7 \pm \sqrt{7}$. It is therefore likely that two identical experiments will see 10 and 4 correlating rays, respectively. The first one would see a strong correlation signal (10 events at 2 expected with the hypothesis of no correlations; probability $p \sim 5 \times 10^{-5}$ ), the second one would see a signal compatible with the uniform background (4 events at 2 expected, $p \sim 14 \%$ ).

It is worth stressing that, in this situation, the final conclusion would be that the correlation exists, because the first of the two experiments is incompatible with uniform background (while both are compatible with the signal). Thus, at present small statistics one should expect that some of the experiments see no correlations. Such fluctuations indeed exist, e.g., in the autocorrelation signal [4, 8].

\section{BL LAC SAMPLES}

There were three samples of BL Lacs discussed in the literature, all three drown from the catalog of quasars and active galactic nuclei [15]: 
(1) The set of 22 most powerful BL Lacs, Ref. [11. This set was found by adjustment of cuts on magnitude and radio flux in the catalog of confirmed BL Lacs using the requirement of the best correlation signal with the most clustered subset of AGASA and Yakutsk cosmic ray events. Neutral primaries were assumed.

(2) The set of 14 BL Lacs which contains potentially $\gamma$-ray-loud objects, Ref. [13. This subset was obtained by the positional cross-correlation of confirmed BL Lacs with the catalog of EGRET sources [16]. Both assumptions of neutral and charged primary particles were tested using the most clustered subset of AGASA and Yakutsk events.

(3) The set of all confirmed BL Lacs with the single cut on visual magnitude, mag $<18$, Ref. [12. This set of BL Lacs contains 156 objects. Both neutral and charged primaries were tested using the whole available set of AGASA events, $E>4 \times 10^{19} \mathrm{eV}$. Better correlations were found under the assumption of positively charged primary particles.

Note that the subsets (1-3) are not independent. BL Lacs which correlate with the UHECR in the set (1) are also present in the set (2). The cut on magnitude, mag $<18$, being the single cut for the set (3), is also imposed in the set (1) among other cuts.

\section{PROCEDURE}

Our analysis is based on the calculation of the angular correlation function by means of the algorithm described in Ref. 11. The statistical significance of correlation is estimated by testing the hypothesis that the UHECR and BL Lacs are uncorrelated. This is done as follows. For a given set of sources and the angle $\delta$, we count the number of pairs "cosmic ray - source" separated by the angular distance less or equal $\delta$, thus obtaining the "data count". We then replace the real data by a randomly generated Monte-Carlo set of cosmic rays and calculate the number of pairs in the same way, thus obtaining the "Monte-Carlo count". We repeat the latter procedure many times noting cases when the Monte-Carlo count equals or exceeds the data count. The number of such cases divided by the total number of tries gives the probability $P(\delta)$ which characterizes the significance of correlations at a given angular scale $\delta$. The smaller is this probability, the stronger (more significant) is the correlation.

The Monte-Carlo events are drown from the isotropic distribution according to the acceptance of a given UHECR detector. For the HiRes experiment in the stereo mode we use the zenith-angle and azimuth- angle distributions given in Ref. 17] and the sidereal time distribution published in Ref. [18].

The probability $P(\delta)$ depends on the choice of $\delta$. The optimal value of $\delta$ is clearly the one where the $e x$ pected signal is strongest (e.g., expected $P_{\mathrm{th}}(\delta)$ is lowest). In the case of point sources and neutral primary particles the shape of $P_{\mathrm{th}}(\delta)$ can be calculated by the following Monte-Carlo simulation. We start by generating a cosmic ray set which is artificially correlated with BL Lacs. This is achieved by replacing a given (small) number of events in a random set by the events correlated with randomly chosen BL Lacs. To generate an event correlated with the BL Lac we assume the 2dimensional Gaussian distribution normalized in such a way that the circle of the radius $0.6^{\circ}$ around the BL Lac contains $68 \%$ of events. The resulting correlated set is then treated as the real data, i.e., the probability $P(\delta)$ is calculated. The bin-by-bin median values of the probabilities obtained for many different sets give $P_{\mathrm{th}}(\delta)$. In the real data, following Ref. [19, we measure correlations at that value of $\delta$ where $P_{\mathrm{th}}(\delta)$ is minimal.

\section{RESULTS}

In Figures 1-3 we present the expected (thin line) and observed (thick line) $P(\delta)$ for the HiRes dataset of UHECR and three samples of BL Lac objects described in Section [3. There are no significant correlations with the subsets of 22 and 14 BL Lacs. In both cases only two BL Lacs contribute to data counts within 1 degree. In the subset of 22 BL Lacs those are TXS 0751+485 and OT 465 (the latter object was among 5 BL Lacs which contribute to correlations in Ref. [11). In the subset of $14 \mathrm{BL}$ Lacs two contributing objects are PKS $1604+159$ and $1 \mathrm{ES} 1959+650$. It is worth mentioning that $1 \mathrm{ES} 1959+650$ is a confirmed TeV source and contributes to correlations in Ref [13].

The set of 156 BL Lacs with mag $<18$ shows rather strong correlation. We list in Table 1 the cosmic rays and BL Lac's separated by less than $1^{\circ}$. The expected probability $P_{\mathrm{th}}(\delta)$ has minimum at $\delta=0.8$. At this value of $\delta$ the real data give the probability $P(0.8)=4 \times 10^{-4}$. The data count is 11 , while the Monte-Carlo expectation due to random uncorrelated background is $\sim 3$. This corresponds to about 8 events from sources in the overall HiRes data-sample of 271 events. Thus, a $\sim 3 \%$ fraction of neutral particles in the total cosmic ray flux may be sufficient to explain this correlation. This is consistent with a fraction of clustered events observed by AGASA at these energies [20]. 


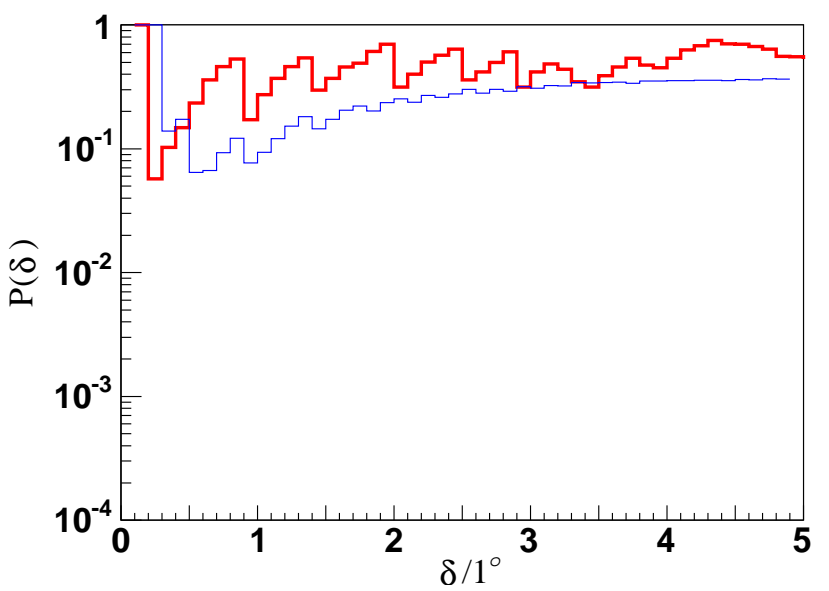

FIG. 1. $P(\delta)$ for the set of 22 BL Lacs and HiRes stereo events. The thick line shows data, the thin line shows $P_{\mathrm{th}}(\delta)$ obtained in the Monte-Carlo simulations in which 2 events are from the BL Lac sources.

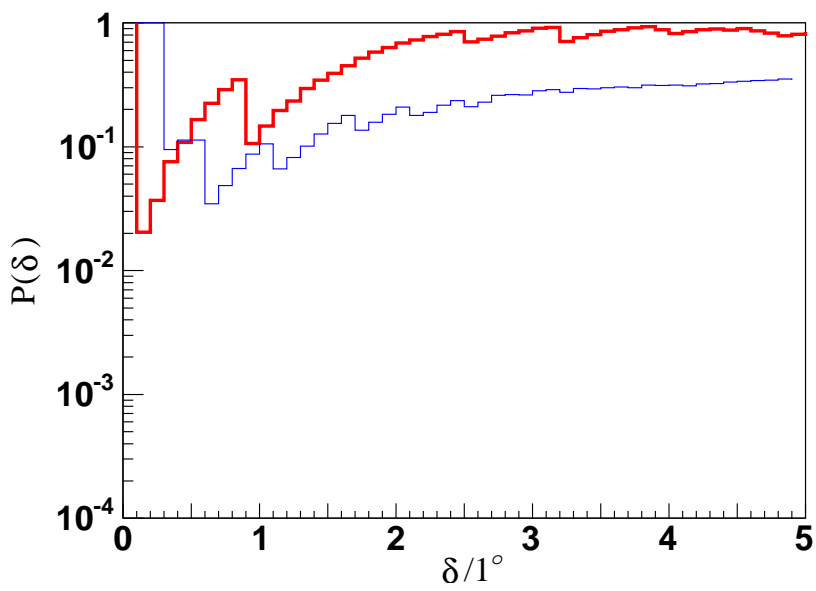

FIG. 2. $P(\delta)$ for the set of 14 BL Lacs and HiRes stereo events. The thick line shows data, the thin line shows $P_{\text {th }}(\delta)$ obtained in the Monte-Carlo simulations in which 2 events are from the BL Lac sources.

\section{CONCLUSIONS}

We have assumed - as a "null-hypothesis" — that BL Lacs and the new HiRes stereo data are uncorrelated. We have tested this hypothesis for 3 particular subsets of BL Lacs studied previously and found an excess of pairs "BL Lac - cosmic ray" in one of them, which would occur with the probability $4 \times 10^{-4}$ for a random set of UHECR. If these sets were independent, the final significance would be the best probability found multiplied by the number of tries. Since the samples are

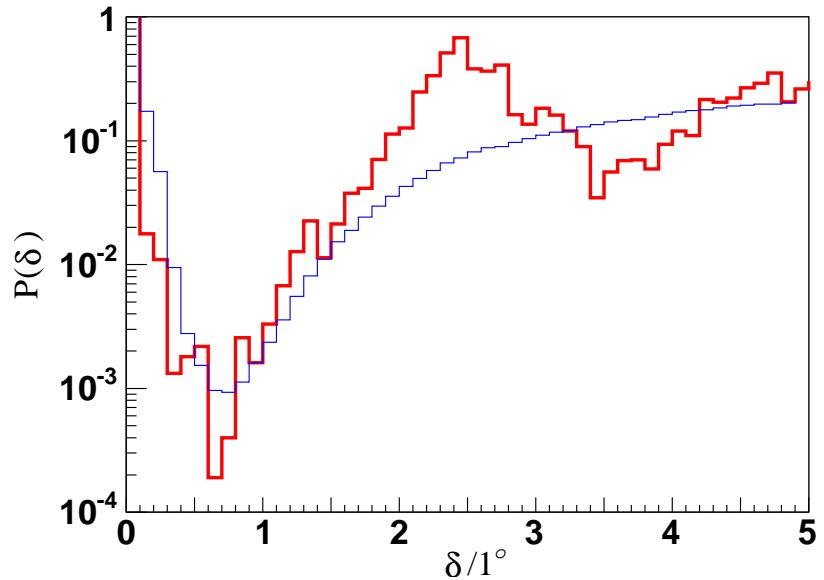

FIG. 3. $P(\delta)$ for the set of $156 \mathrm{BL}$ Lacs and HiRes stereo events. The thick line shows data, the thin line shows $P_{\text {th }}(\delta)$ obtained in the Monte-Carlo simulations in which 9 events are from the BL Lac sources.

\begin{tabular}{|c|c||c|c|}
\hline \multicolumn{2}{|c||}{ Cosmic ray } & \multicolumn{2}{c|}{ BL Lac } \\
\hline$\alpha$, deg. & $\delta$, deg. & name & $z$ \\
\hline 17.8 & -12.5 & RBS 0161 & 0.234 \\
48.5 & 5.8 & RX J03143+0620 & $?$ \\
118.7 & 48.1 & TXS 0751+485 & $?$ \\
123.8 & 57.0 & RX J08163+5739 & $?$ \\
137.2 & 33.5 & Ton 1015 & 0.354 \\
162.6 & 49.2 & MS 10507+4946 & 0.140 \\
169.3 & 25.9 & RX J11176+2548 & 0.360 \\
209.9 & 59.7 & RX J13598+5911 & $?$ \\
226.5 & 56.5 & SBS 1508+561 & $?$ \\
229.0 & 56.4 & SBS 1508+561 & $?$ \\
253.7 & 39.8 & RGB J1652+403 & $?$ \\
265.3 & 46.7 & OT 465 & $?$ \\
300.2 & 65.1 & 1ES 1959+650 & 0.047 \\
\hline
\end{tabular}

Table 1. Cosmic rays and BL Lac's from the sample of 156 objects separated by less than $1^{\circ}$. Note that SBS $1508+561$ correlates with two rays.

not independent, the penalty factor is smaller. In any case, the null-hypothesis is rejected with the probability $\sim 10^{-3}$. Our analysis thus confirms the association of UHECR with BL Lacs.

Only one of the three examined subsets of BL Lacs - the largest one - shows significant correlations. This may indicate that those BL Lacs which are sources, have similar and small luminosity in UHECR. This conclusion is also consistent with the statistics of the clus- 
tering of UHECR events, which suggests few hundred sources currently contributing to the UHECR flux, see Refs. 21] 22, 10].

The observation of correlations at angles which are much smaller than the typical deflection of a charged UHECR particle in the Galactic magnetic field suggests that (at least) a few percent of UHECR are neutral.

The authors are indebted to K. Belov, V. Berezinsky, M. Libanov, A. Neronov, D. Semikoz and T. Weiler for interesting discussions and useful comments. This work was supported in part by the INTAS grant 0351-5112 (D.G., P.T. and S.T.), by the RFBR grant 0202-17398 (D.G. and S.T.), by the grants of the President of the Russian Federation NS-2184.2003.2, MK2788.2003 .02 (D.G.), MK-1084.2003.02 (S.T.), by the grants of the Russian Science Support Foundation (D.G. and S.T.), by the fellowships of the "Dynasty" foundation (awarded by the Scientific board of ICFPM; D.G. and S.T.) and by the Swiss Science Foundation, grant 20-67958.02 (P.T.).

1. V. S. Berezinsky et. al., Astrophysics of cosmic rays, Amsterdam: North Holland (1990).

2. J. P. Rachen and P. L. Biermann, Astron. Astrophys. 272 (1993) 161.

3. V. Berezinsky, A. Z. Gazizov and S. I. Grigorieva, arXiv:hep-ph/0204357

4. M. Takeda et al. (AGASA Collaboration), Astrophys. J. 522, 225 (1999).

5. P. G. Tinyakov and I. I. Tkachev, JETP Lett. 74 (2001) 1 [Pisma Zh. Eksp. Teor. Fiz. 74 (2001) 3].

6. C. B. Finley and S. Westerhoff, arXiv: astro-ph/0309159

7. R. U. Abbasi et al. [The High Resolution Fly's Eye Collaboration], arXiv: astro-ph/0404366

8. R. U. Abbasi et al. [The High Resolution Fly's Eye Collaboration], arXiv: astro-ph/0404137

9. H. Yoshiguchi, S. Nagataki and K. Sato, arXiv:astro-ph/0404411

10. M. Kachelriess and D. Semikoz, arXiv:astro-ph/0405258

11. P. G. Tinyakov and I. I. Tkachev, JETP Lett. 74, 445 (2001) [Pisma Zh. Eksp. Teor. Fiz. 74, 499 (2001)].

12. P. G. Tinyakov and I. I. Tkachev, Astropart. Phys. 18, 165 (2002).

13. D. S. Gorbunov et al., Astrophys. J. 577 (2002) L93.

14. A. V. Uryson, Results of identification of UHECR sources, Proc. 27th ICRC (Hamburg), 2001, 551.

15. M. P. Véron-Cetty and P. Véron, ESO scientific report (2000); M. P. Véron-Cetty and P. Véron, Astron. Astrophys. 374 (2001) 92.

16. R. C. Hartman et.al., Astrophys. J. Suppl. 123 (1999) 79 .
17. R. W. Springer, Stereo spectrum of UHECR showers at the HiRes detector, presentation at the 29th ICRC, Tsukuba, 2003.

18. B. T. Stokes, C. C. H. Jui and J. N. Matthews, Astropart. Phys. 21 (2004) 95.

19. P. Tinyakov and I. Tkachev, arXiv:astro-ph/0301336

20. M. Takeda for the AGASA Collaboration Clusters of Cosmic Rays above $10^{19} \mathrm{eV}$ observed with AGASA, Proc. of 27th ICRC (Hamburg) 1 (2001) 341.

21. S. L. Dubovsky, P. G. Tinyakov and I. I. Tkachev, Phys. Rev. Lett. 85 (2000) 1154.

22. P. Blasi and D. De Marco, Astropart. Phys. 20 (2004) 559 arXiv:astro-ph/0307067. 